\title{
Analysis of Chemical Kinetics Simulation of the Removal of Formaldehyde in Dielectric Barrier Discharge
}

\author{
Yaping Li \\ Ankang University, Ankang , 725000, China
}

Keywords: dielectric barrier discharge, removal of $\mathrm{HCHO}$, chemical kinetics simulation

\begin{abstract}
This paper mainly analyzes chemical kinetics simulation of the removal of formaldehyde (HCHO) in atmospheric dielectric barrier discharge, builds a time-space average model, and verifies relevant results through experiment. Changes in concentration of various substances with discharge time are analyzed through chemical experiment. In simulated atmospheric environment, HCHO is mainly removed by free radicals of oxygen ions and hydroxyl ions, among which, hydroxyl ions have stronger force. In chemical kinetics simulation, the first electronically excited state of nitrogen molecules is stressed, and collision of these molecules with oxygen molecules and water molecules has an influence on the concentration of oxygen ions and hydroxyl ions. In nitrogen environment, the first electronically excited state of nitrogen molecules is the main substance for removal of $\mathrm{HCHO}$. This paper discusses about the influence of initial concentration of HCHO and gas flow etc on specific energy consumption of removal of $\mathrm{HCHO}$ and molar ratio of $\mathrm{CO}_{2} / \mathrm{CO}$ among experimental products. When initial concentration of $\mathrm{HCHO}$ is inversely proportional to specific energy consumption of removal of $\mathrm{HCHO}$ and input energy density is lower than $60 \mathrm{~J} / \mathrm{L}$ and concentration of $\mathrm{HCHO}$ is low, molar ratio of $\mathrm{CO}_{2} / \mathrm{CO}$ among experimental products is high.
\end{abstract}

\section{Introduction}

Dielectric barrier discharge is a plasma technology that is commonly used for generating atmospheric pressure cold, and is increasingly concerned at present because of wide application in researches on removing pollutants from air. Famous chemist Sun and other scientists report the application of dielectric barrier discharge in experiment of removing sulfur dioxide and nitric oxide from air. Through a large amount of experimental data, the influence of input energy density and pulse frequency on removal efficiency of sulfur dioxide and nitric oxide is analyzed and corresponding law is obtained. Dorai et al report chemical kinetics experiment of removal of soot and nitrogen oxides in dielectric barrier discharge, and describe in detail reactions between nitrogen oxides and positive ions and hydroxyl free radical carbon oxides. While another group of people find out principle of removing $\mathrm{HCHO}$ by "direct electron collision" combined with "free radical reaction" when conducting experiment of removing $\mathrm{HCHO}$ in dielectric barrier discharge, but this principle does not consider about the function of the first electronically excited state of nitrogen molecules.

Experiment of chemical kinetics simulation analysis of removal of $\mathrm{HCHO}$ in dielectric barrier discharge in this paper not only considers about the function of positive ions and hydroxyl ion free radicals, but also uses the feature of nitrogen molecules namely the first electronically excited state. Besides, this paper also analyzes the influence of initial concentration of HCHO, gas flow, comparative energy consumption, and molar ratio of $\mathrm{CO}_{2} / \mathrm{CO}$ among experimental products on the experiment, and thus verifies simulation results.

\section{Basic theories in experiment}

(1) Describe chemical kinetics model by mathematics

The nature of dielectric barrier discharge is caused by lots of small fast pulse discharge channels in air, and these channels lead to various chemical reactions during discharge. To make these chemical phenomena specific and digital, highly nonlinear differential equations containing resolution time and space during the entire experiment shall be solved. But, during the actual 
experiment, voltage and current when discharging are in extremely unstable oscillation, and thus it is difficult to obtain accurate electron density with both time and space resolution. So, researchers build a 0-dimensional kinetics model with average time and space resolution, based on the following three preconditions:

1. During discharge, time distribution and space distribution of electron density are uniform;

2. There is a certain functional relationship between electron concentration and input energy density, and the functional relation is: $n_{\mathrm{e}}=\beta \mathrm{E}_{\mathrm{n}}^{\mu}$. Where, $\beta$ and $\mu$ are obtained by comparing real experiment and simulation results, i.e. to obtain rate of change of $\mathrm{HCHO}$ removal rate when energy density changes under experimental environment with the concentration of $\mathrm{HCHO}$ system of 80 and gas flow of $90 \mathrm{ml} / \mathrm{min}$. That is to say, in the experiment, removal rate of each HCHO molecule is closely related to input energy density in experiment and electron density in simulation. Thus, the final conclusions are: $\beta=8.2 \times 10^{5}, \mu=0.6$.

3. After gas in discharge area flows out, ions may still exist and continue to have chemical reactions, but the electron density is 0 .

In this reaction system, substances involved in elementary reactions can be expressed by a rate equation, and can be summarized as an initialization issue of differential equations associated with influence of concentration of relevant species on the experiment. Thus, study on the change law of concentration of different substances in different time, and build chemical reaction kinetics differential equations with variables of concentration of all substances occurred in experimental system, and solve by corresponding computer language programming. In the equations, number of species is order of coupling rate equations, the basic form of which is:

$D n_{\mathrm{i}} / \mathrm{d}_{\mathrm{t}}=\sum-\mathrm{k}_{\mathrm{ij}} \mathrm{n}_{\mathrm{i}} \mathrm{n}_{\mathrm{j}}+\sum \mathrm{k}_{\mathrm{pq}} \mathrm{n}_{\mathrm{p}} \mathrm{n}_{\mathrm{q}}$

In this equation system, reaction rate constant often varies greatly, and decay rate of different substances varies greatly, and change rate may be sometimes unstable, which brings inconvenience to numerical integration statistics. Description of the change process shall be determined under very short time step, so as to keep the entire numerical calculation stable; however, time step shall be long so that integration area could reach stable, which leads to rigid contradiction. Treanor's method shall be taken to solve this problem, as it adds a value with fast varying component in approximant and thus solves problem in polynomial approximation rigid equation.

(2) Plasma chemical reactions

To fully reproduce chemical kinetics process of removal of $\mathrm{HCHO}$ in dielectric barrier discharge, the built chemical model contains a total of 20 substances, including excited state of various molecules, atoms, free radicals and substances, and generates 116 chemical reactions, which can be divided into two categories, namely reactions due to collision between electrons and gas molecules and reactions due to collision among active substances. In collision reactions between electrons and gas molecules, constants of dissociation and stimulation reaction rates are obtained by solving equations of electron energy and corresponding collision cross-sections. Here, several plasma chemical reactions commonly used in experiment are listed as follows:

$$
\begin{aligned}
& \mathrm{HCHO}+\mathrm{N}_{2}\left(\mathrm{~A}^{3} \sum_{\mathrm{u}}^{+}\right) \rightarrow \mathrm{H} \mathrm{CO}^{-}+\mathrm{H}^{+}+\mathrm{N}_{2} \\
& \mathrm{HCHO}+\mathrm{O} \mathrm{H}^{-} \rightarrow \mathrm{H} \mathrm{C} \mathrm{O}^{-}+\mathrm{H}_{2} \mathrm{O} \\
& \mathrm{HCHO}+\mathrm{O}^{2-} \rightarrow \mathrm{HCO}^{-}+\mathrm{O} \mathrm{H}^{-}
\end{aligned}
$$

\section{Experimental operation}

The following equipment shall be configured in this experiment: a coaxial electrode reactor, a quartz tube with the center of $1.6 \mathrm{~mm}$ stainless steel bar, $4 \mathrm{~mm}$ inner diameter and $1 \mathrm{~mm}$ wall thickness, connect this quartz tube to the output end of AC high-voltage power supply, and wrap a layer of stainless steel mesh outside the tube as grounding electrode; $50 \mathrm{~Hz} 0-40 \mathrm{KV}$ continuously adjustable AC high-voltage power supply; nearly 100\% pure oxygen and nitrogen. Experimental procedures are that purified water passes through nitrogen environment, flows through a water bath temperature-controlled bubble-type bottle, and finally enters the reactor; in experimental equipment on the other side, nitrogen carries trioxymethylene vapor in a water bath temperature-controlled and 
generates $\mathrm{HCHO}$ by catalytic decomposition in a $160^{\circ} \mathrm{C}$ environment. Concentration of $\mathrm{HCHO}$ generated in the experiment can be adjusted by water bath temperature in the experiment, and can be examined and tested online by SRI-8610C gas chromatograph. Concentration of $\mathrm{CO}$ and $\mathrm{CO}_{2}$ needed in the experiment will be tested by four components of infrared absorption gas analyzer. The entire experiment shall be conducted under atmospheric pressure and ambient temperature, and the components of simulated air, initial concentration of $\mathrm{HCHO}$ and gas flow shall be controlled.

\section{Analysis of experimental results}

(1) Change rate of concentration of active substances in the experiment

According to the above experiment, the author obtains a series of data, and shows changes in concentration of oxygen ions, hydroxyl free radicals, water molecules, ozone molecules and HCHO with time in charts in the form of coordinate axis, in which time zero begins when gas enters the discharge area. Substances used in the experiment can be divided into two categories because of different paths of generation: initial free radicals, such as oxygen ions and hydroxyl free radicals; secondary free radicals, such as hydrogen peroxide free radicals. It can be known from data analysis that oxygen ions and hydroxyl free radicals play a decisive role in removal of $\mathrm{HCHO}$, and nitrogen molecules play a very important role and arouse more concern in plasma chemical reaction kinetics researches because of approximately two-second radiation lifetime and extremely high energy. As can be found in experimental comparison, the first electronically excited state of nitrogen molecules plays a significant promoting role in generation of oxygen ions and hydroxyl free radicals. When gas enters discharge area, concentration of the first electronically excited state of nitrogen molecules increases straightly; when gas leaves the discharge area, concentration of the first electronically excited state of nitrogen molecules decreases straightly, while maintains in a certain range during middle period of time. Such phenomena occur because generation consumption reaction rate constant of the first electronically excited state of nitrogen molecules is generally large with almost the same generation and consumption rates during discharge, and thus concentration of gas in discharge area maintains at a stable state.

In the experiment, secondary free radicals such as ozone molecules and hydrogen peroxide free radicals can further have chemical reaction generation through initial free radicals and have great influence on carbon dioxide. As concentration of free radicals is low, very little HCHO will be reduced during discharge. After a certain time of experiment when the maximum concentration of oxygen ions is reached, the concentration of hydroxyl free radicals continues to rise, and then the concentration of $\mathrm{HCHO}$ changes very rapidly until reaching discharge area. Then, when the concentration of free radicals decreases increasingly quick, concentration of $\mathrm{HCHO}$ will not change. During the entire experiment, it can be seen from analysis of change rate of substance concentration that hydroxyl free radicals play a greater promoting role in removal of HCHO than oxygen ions. To verify this conclusion, scientists make two types of simulation calculation, remove reactions of oxygen ions and $\mathrm{HCHO}$ first in experiment and conduct statistics of removal rate of $\mathrm{HCHO}$ in experimental results; then, remove reactions of hydroxyl free radicals and HCHO in the second experiment and conduct statistics of removal rate of HCHO in experimental results. Finally, it is found through comparison that the former is significantly larger than the latter, and the sum of both is far smaller than HCHO removal rate in former experiment, because reactions of oxygen ions and $\mathrm{HCHO}$ in experiment will generate hydroxyl free radicals, and the concentration of hydroxyl free radicals will be reduced without such reactions. In the above two types of simulation calculation, reactions of oxygen ions and $\mathrm{HCHO}$ molecules will generate $20 \% \mathrm{HCHO}$ removal rate, while those of hydroxyl free radicals and HCHO molecules will generate $62.3 \%$ removal rate. In general, hydroxyl free radicals and oxygen ions play only one third of role in removal of HCHO.

(2) Specific energy consumption and product selection

Different conditions are taken for chemical kinetics simulation experiment of removal of HCHO in dielectric barrier discharge, and change law of specific energy consumption of HCHO removal with energy density is found through data analysis, and specific energy consumption of HCHO 
removal decreases gradually when input energy density is reduced; and specific energy consumption of $\mathrm{HCHO}$ removal will gradually increase when energy density increases. When input energy density is quite low, specific energy consumption changes exponentially. So, in electron collision reaction, discharge energy will be utilized more efficiently. In nitrogen environment, removal rate of $\mathrm{HCHO}$ is quite low, and specific energy consumption at this time is higher than in simulated atmospheric environment on equal conditions. When initial concentration of HCHO is quite high, $\mathrm{HCHO}$ has low specific energy consumption, as various substances that react with $\mathrm{HCHO}$ in experiment are fully used; however, when its initial concentration is low, these active substances have not enough time to collide with $\mathrm{HCHO}$ and are consumed with other substances.

Products of experiment of low-temperature plasma $\mathrm{HCHO}$ removal are $\mathrm{CO}, \mathrm{CO}_{2}$ and $\mathrm{H}_{2} \mathrm{O}$. In nitrogen environment, due to lack of oxidizing substances, molar ratios of $\mathrm{CO}_{2}$ and $\mathrm{CO}$ are zero. However, in the above conclusions, change rate of HCHO remains almost unchanged in different gas flows because of the same input energy density. In the entire experiment, among products of pure experiment of $\mathrm{HCHO}$ removal of dielectric barrier discharge, $\mathrm{CO}$ has higher selectivity than $\mathrm{CO}_{2}$. So, other methods shall be used to improve the selectivity of $\mathrm{CO}_{2}$, such as adding catalyst in reactor.

\section{Conclusions}

This experiment builds a simple reaction system, and analyzes chemical kinetics of HCHO removal in dielectric barrier discharge, chemical kinetics model by mathematics and plasma chemical reactions. In simulated atmospheric environment, by analyzing change law of the concentration of main substances in discharge experiment with time, it is found that oxygen ions and hydroxyl free radicals determine the removal of $\mathrm{HCHO}$, and the first electronically excited state of nitrogen molecules improves the concentration of oxygen ions and hydroxyl free radicals in experiment; while in nitrogen environment, the first electronically excited state of nitrogen molecules is decisive substance of HCHO removal, and specific energy consumption of HCHO removal decreases first and then increases with the increase of input energy density. There is little difference between experimental results in this paper and simulation results with a deviation range less than $20 \%$. In experiment, the relationship between electron density and parameters of dielectric barrier discharge should be more accurate, and function expression of electron density and input energy density is only for the convenience of simulation. In addition, although electron density is not evenly distributed in time and space in reality, this simple model still provides certain help for study on chemical kinetics mechanism in dielectric barrier discharge.

\section{References}

[1] Zhang Jing, Lu Fugong, Xu Yong, Yang Xuefeng, Zhu Aimin. Chemical Kinetics Simulation of the Removal of Formaldehyde in Dielectric Barrier Discharge [J]. Acta Physico-Chemica Sinica, 2010, 09: 1425-1431.

[2] Zhang Jing. Chemical Kinetics Simulation of the Removal of Formaldehyde in Dielectric Barrier Discharge [D]. Dalian University of Technology, 2011.

[3] Wang Lina. Numerical Simulation of Plasma $\cdot \mathrm{OH}$ and $\mathrm{HO}_{2} \cdot$ Free Radicals in Dielectric Barrier Discharge [D]. Dalian University of Technology, 2010.

[4] Wang Xiaojing. Atmospheric Pressure Dielectric Barrier Discharge Multi-needle-Coaxial Reactor Structural Optimization and Degradation HCHO Experimental Study [D]. Chongqing University, 2012.

[5] Ding Huixian. Study on Low-temperature Removal of HCHO in Gas by Plasma Coordinated with Catalyst under Atmospheric Pressure [D]. Dalian University of Technology, 2009. 